\title{
Image Enhancement of Low Resolution Satellite Image based on Texture and Morphological Features
}

\author{
Snehal Godage \\ Department of Electronics and \\ Telecommunication Engineering, \\ N B Navale Sinhgad College of \\ Engineering \\ Kegaon, Solapur, 413255
}

\author{
S. P. Sagat \\ Professor \\ Department of Electronics and \\ Telecommunication Engineering, \\ N B Navale Sinhgad College of \\ Engineering \\ Kegaon, Solapur, 413255
}

\author{
A. D. Shinde \\ Professor \\ Department of Electronics and \\ Telecommunication Engineering, \\ N B Navale Sinhgad College of \\ Engineering \\ Kegaon, Solapur, 413255
}

\begin{abstract}
In a modern and industrial world, image processing plays a vital role to make the applications more smart compare to the present systems. Image Enhancement, a major term in image processing industry, which is more innovative and crucial task in digital image processing domain. The main intention of the digital image processing and enhancement scheme is to portrait the visual inspection of the image in better contrast with proper sharpness and brightness. The Satellite Image Processing scheme is most essential image processing task, which illustrates the processing of converting complex and blurred view of images into better clarified view to the user. The term morphological feature analysis illustrates the processing of extracting the features of satellite images as well as enhancing the clarity of the respective image in better manner. The proposed approach of satellite image processing clearly demonstrates the process of textural and morphological features of respective image and provides better visual clarity to understand the input image with proper level of accuracy. In the proposed approach, some classification schemes are taken care for processing the image with better clarity, such as Support Vector Machine [SVM], Artificial Neural Network [ANN] and so on. For all the entire work clearly demonstrates the process of manipulating the satellite image processing to provide better quality of images with more contrast as well as accuracy in result.
\end{abstract}

\section{Keywords}

Digital Image Processing, Satellite Images, Feature Extraction, Morphological Analysis, Image Enhancement

\section{INTRODUCTION}

Image Processing, a major domain in Information Technology industry, which deals the processing of digital images as well as providing different kinds of solutions to corresponding users. The processing of contrast analysis, feature extraction, resolution checking as well as enhancement of satellite images requires more concentration in this image processing industry.
Specifically, the image enhancement schemes are supervised to change the band-intensities as well as reduce the noisy pixels, which save the specific required data of image. LinearContrast Manipulation, Edge Smoothing, Transformation, Histogram Feature Evaluation, Filtration Schemes, Decomposition and composition schemes and so on. Many classical techniques are available to process the satellite images such as: Transformation schemes like FourierTransformation, Wavelet-Decomposition, Domain Image Processing and many more. The major objective of all these quoted image processing techniques are improving the visual quality of the respective input image as well as enhancing the clarity level within defined and reduced timings. The proposed approach is also guaranteed those things to achieve more clarity of the satellite image by means of applying the textural and morphological feature analysis scheme over digital image processing domain to improve the view of satellite images with low-level of toughness as well as achieving high level of accuracy. The proposed approach constitutes a data preparation phase just before the feature extraction. It attempts to improve the quality of the textural/morphological characteristics while retaining the computational burden in low levels.

\section{PROPOSED SYSTEM OBJECTIVE}

The main objective of the proposed system is to provide good quality and better view of satellite images, which is not clear and illuted in nature. This is done by using the following procedures. Initially the input of three band images is processed by reprocessing technique such as converting the input image into gray scale manner as well as resizes the pixels into base nature like 256X256. The next step of processing is based on histogram based feature analysis, which is performed individually for all bands of the input image. In this stage a new replication is produced as well as which is made by the powerful classification technique called Support Vector Machine [SVM]. 


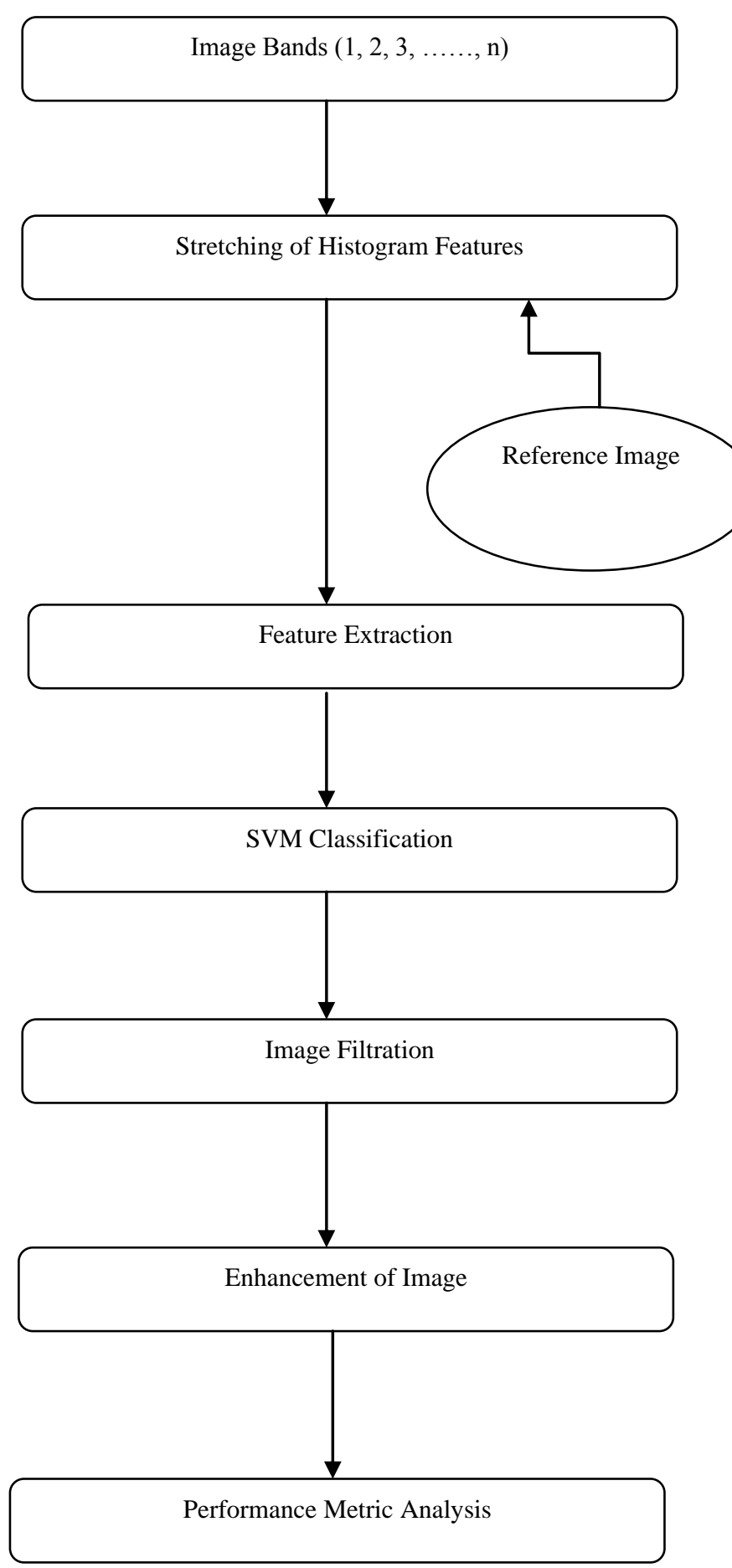

Fig.1. Block Diagram of Proposed System

The next stage of processing is to adjusting the pixels and improves the contrast of the input image to make it so perfect for the future processing. The next step is to extract the color features of the images based on Red, Green and Blue [RGB]. The next step is to measure the performance and accuracy level of the input image with balanced pixel manipulations. Finally, the process ends with SSL [Soil Sealing Layer], which is used for the data set and it is from European areas and it is useful to provide the information of degree of soil sealing in spatial resolution.

\section{SCOPE OF THE PROPOSED SYSTEM}

Most of the previously mentioned procedures are proposed for enhancing the visual perspective of the satellite images and as a rule includes physical attribute fixing. The prerequisites of this system, as outlined further as well as it goes below-down, force a completely computerized approach. The satelliteimages can be viewed as a kind of valuable data/information in 3D remaking, building position acknowledgment, and tallness estimation. This work incorporates tests with versatile-histogram alteration, utilization of various reference layers, furthermore, use of radiometric include choice and change methods.

\section{IMAGE FEATURE ANALYSIS}

The textural estimations are assessed through the past researcher Haralick"s-measure for the force differentiates between a pixel and its neighbors. The components [quantization, length, position and shape] and the administrators like Fuzzy'-creation are characterized in the delivered textural layer is known as 'PANTEX'. With respect to morphological highlights, it incorporated into tests an as of late presented list named Morphological Building Index $[\mathrm{MBI}]$. It is a very precise marker that considers the attributes of structures [splendor, estimate, differentiation, Directionality and shape] by incorporating multi-scale and multi-directional morphological administrators. Note that both PANTEX and MBI are programmed records and their operation did not depend on factual learning and preparing tests. It changes over the multi-band satellite images into eight bit gray scale. Moreover, the gray level images are appropriate to work with morphological administrators. The complexity change should be possible either the image transformation to gray scale by methods for a reasonable phantom band mix or by adjusting the histogram of the image densities.

\section{CONTRAST ENHANCEMENT}

The final procedural step is the alteration of the image histogram. Now, there are two-investigate situations. The initial image (X1) is changed over to gray scale and after that, the contrast modification happens. The histogram (X2) modification is done independently for each band of the unique image (Multichannel Histogram Extending) and after that, the groups are converged to shape a gray scale image.

The nature of image is enhanced by applying contrast upgrade, utilizing histogram modification for each layer in particular as multi-channel histogram extending and consolidating it to get the single band image. To change the printed qualities Gaussian spiral capacity was utilized. The surface is resolved interestingly by the thickness of the distinguished corners. The morphological trademark is controlled by the progressive utilization of basic procedure of separating between objects recognized at disparate scales. The yield is to extricate the component of the low determination satellite image, first by combining the groups and after that applying the histogram extending.

\section{RELATED STUDY}

In the year of 2010, the authors "S. Kim, W. Kang, E. Lee, and J. Paik" proposed a system titled "Wavelet-domain color image enhancement using filtered directional bases and frequency-adaptive shrinkage" in that they described such as: this system displays a novel wavelet-space shading picture upgrade utilizing separated directional bases and recurrence versatile shrinkage. Most customary commotion lessening 
strategies tend to over-smother high-recurrence points of interest. For beating this issue we initially break down the information picture into level and edge areas, and expel clamor utilizing the alpha guide processed from wavelet change coefficients of $\mathrm{LH}, \mathrm{HL}$, and $\mathrm{HH}$ groups. In the wake of evacuating clamor in the level locale, we additionally expel commotion in edge districts by adaptive contracting wavelet coefficients in light of the entropy. Besides, we introduce another directional change utilizing wavelet premise and Gaussian low pass channels. The wavelet coefficients of edge locales are converse changed by utilizing the separated wavelet bases. Exploratory outcomes demonstrate the proposed calculation can decrease clamor without losing sharp points of interest and is reasonable for business minimal effort imaging frameworks, for example, computerized cameras, CCTV, and observation framework.

In the year of 2010, the authors "A. R. Gillespie, A. B. Kahle, and R. E. Walker" proposed a system titled "Color enhancement of highly correlated images. I. Decorrelationand HSI contrast stretches" in that they described such as: Customary upgrades for the shading showcase of multispectral pictures depend on autonomous difference adjustments or "extends" of three info pictures. This approach isn't viable if the picture channels are very related or if the picture histograms are firmly Bimodal or more perplexing. Any of a few systems that tend to "extend" shading immersion while leaving tone unaltered may better use the full scope of hues for the show of picture data. Two theoretically extraordinary upgrades are examined: the "decorrelation extend", in view of foremost segment (PC) examination, and the "extend" of "shade"- "immersion"- force (HSI) changed information. The PC change is scenesubordinate; however the HSI change is invariant. Cases of pictures upgraded by ordinary straight extends, decorrelation extend, and by extends of HSI changed information are analyzed. Schematic variety charts or two-and threedimensional histograms are utilized to represent the "decorrelation extend"technique and the impact of the diverse improvements.

In the year of 2010, the authors "H.Demirel, C. Ozcinar, and G. Anbarjafari" proposed a system titled "Satellite image contrast enhancement using discrete wavelet transform and singular value decomposition" in that they described such as: another satellite picture differentiate upgrade method in light of the discrete wavelet change (DWT) and particular esteem disintegration has been proposed. The procedure breaks down the info picture into the four recurrence subbands by utilizing DWT and appraisals the solitary esteem network of the lowlow subband picture, and, at that point, it recreates the upgraded picture by applying opposite DWT. The strategy is contrasted and regular picture adjustment strategies, for example, standard general histogram evening out and neighborhood histogram leveling, and in addition cutting edge methods, for example, brilliance protecting dynamic histogram balance and solitary esteem balance. The trial comes about demonstrate the predominance of the proposed strategy over ordinary and cutting edge systems.

In the year of 2013, the authors "G.Srilekha,V.K.Kumar and B. Jyothi"proposed a system titled "Satellite image resolution enhancement using DWT and contrast enhancement using SVD"in that they described such as: this system presented another satellite picture determination and complexity upgrade procedure which depends on the mix of other two system named as discrete wavelet change (DWT) and particular esteem deterioration (SVD). Satellite pictures are utilized as a part of numerous applications, for example, in meteorology, oceanography, angling, farming, ranger service, topography, training, knowledge and fighting. A standout amongst the most essential quality factors in pictures originates from its determination, here this method decays the info picture into the four recurrence sub-groups by utilizing DWT and the high recurrence sub band pictures which originates from DWT have been added, by including the distinction picture of the information picture alongside this strategy likewise gauges the adjusted solitary esteem lattice from the LL sub band of histogram leveled picture and LL sub band of information picture to get splendor improved picture. Keeping in mind the end goal to get the new picture of better difference and determination all these subgroups are joined utilizing backwards DWT. Proposed system is contrasted and ordinary picture balance procedures, for example, general histogram balance (GHE), neighborhood histogram balance (LHE) and furthermore from best in class strategy which is particular esteem evening out (SVE). At that point the test comes about demonstrate the amazingness of the proposed strategy over regular and condition of-craftsmanship systems.

\section{CONCLUSION}

The implemented approach/framework presents image clarity improvement such as enhancing the image as well as enabling the contrast alteration, which assumes a generous part for the extraction of textural and morphological qualities of the input satellite image. With a specific end goal to research and measure the affect ability of those features/highlights against varieties interestingly, a progression of tests were done; distinctive situations were analyzed in regard to the most part the image bands, blend as well as the image histogram change guided by low-determination reference information. For all the entire work is the presentation of a straightforward and generally quick approach that can "accurate" the low determination reference and move it easily in better resolutions and clarity.

\section{REFERENCES}

[1] F. Y. Shih, Image Processing and Pattern RecognitionFundamentals and Techniques. Hoboken, NJ, USA: Wiley, 2010.

[2] R. C. Gonzalez and R. E. Woods, Digital Image Processing, 2nd ed. Englewood Cliffs, NJ, USA: Prentice Hall, 2002.

[3] S. Kim, W. Kang, E. Lee, and J. Paik, "Wavelet-domain color image enhancement using filtered directional bases and frequency-adaptive shrinkage," IEEE Trans. Consum. Electron., vol. 56, no. 2, pp. 1063-1070, May 2010.

[4] A. R. Gillespie, A. B. Kahle, and R. E. Walker, "Color enhancement of highly correlated images. I. Decorrelation and HSI contrast stretches," Remote Sens. Environ., vol. 20, no. 3, pp. 209-235, Dec. 1986.

[5] H. Demirel, C. Ozcinar, and G. Anbarjafari, "Satellite image contrast enhancement using discrete wavelet transform and singular value decomposition," IEEE Geosci. Remote Sens. Lett., vol. 7, no. 2, pp. 333-337, Apr. 2010.

[6] G. Srilekha, V. K. Kumar, and B. Jyothi, "Satellite image resolution enhancement using DWT and contrast enhancement using SVD," Int. J. Eng. Res. Technol. (IJERT), vol. 2, no. 5, pp. 1227-1230, May 2013. 

http://cidportal.jrc.ec.europa.eu/copernicus/services/web viewer/core003

[8] S. Ferri et al., "A new map of the European settlements by automatic classification of $2.5 \mathrm{~m}$ resolution SPOT data," in Proc. IEEE Int. Geosci. Remote Sens. Symp. (IGARSS), Quebec City, QC, Canada, Jul. 2014, pp. 1160-1163.

[9] A. Burger, G. Di Matteo, and P. J. Åstrand, "Specifications of view services for GMES Core_003 VHR2 coverage," European Commission, JRC Tech. Rep., 2012, doi: 10.2788/21898.

[10] M. Pesaresi et al., "A global human settlement layer from optical HR/VHR RS data: Concept and first results," IEEE J. Sel. Topics Appl. Earth Observ. Remote Sens., vol. 6, no. 5, pp. 2102-2131, Oct. 2013.

[11] [Online]. Available: http://www.eea.europa.eu/articles/urban-soilsealing-ineurope

[12] G. Maucha, G. Büttner, and B. Kosztra, "European validation of GMES FTS soil sealing enhancement data," European Environment Agency, Final draft, Jun. 2010.

[13] G. K. Ouzounis, V. Syrris, and M. Pesaresi, "Multiscale quality assessment of global human settlement layer scenes against reference data using statistical learning,' Pattern Recognit. Lett., vol. 34, no. 14, pp. 1636- 1647, Oct. 2013

[14] R. M. Haralick, K. Shanmugan, and I. Dinstein, "Textural features for image classification," IEEE Trans. Syst. Man Cybern., vol. 3, no. 6, pp. 610-621, Nov. 1973.

[15] M. Pesaresi, A. Gerhardinger, and F. Kayitakire, "A robust built-up area presence index by anisotropic rotation-invariant textural measure," IEEE J. Sel. Topics Appl. Earth Observ. Remote Sens., vol. 1, no. 3, pp. 180-192, Sep. 2008

[16] X. Huang and L. Zhang, "A multidirectional and multiscale morphological index for automatic building extraction from mutispectral GeoEye-1 imagery," Photogramm. Eng. Remote Sens., vol. 77, no. 7, pp. 721732,2011

[17] X. Huang and L. Zhang, "Morphological building/shadow index for building extraction from highresolution imagery over urban areas," IEEE J. Sel. Topics Appl. Earth Observ. Remote Sens., vol. 5, no. 1, pp. 161-172, Feb. 2012.

[18] V. Vapnik, Statistical Learning Theory. Hoboken, NJ, USA:Wiley, 1998 